\title{
Human Dynamics: From Human Mobility to Predictability
}

\author{
Albert-László Barabási \\ Center of Complex Networks Research, Northeastern University and Department of \\ Medicine, Harvard University \\ barabasi@gmail.com
}

\begin{abstract}
A range of applications, from predicting the spread of human and electronic viruses to city planning and resource management in mobile communications, depend on our ability to understand human activity patterns. I will discuss recent effort to explore human activity patterns, using the mobility of individuals as a proxy. As an application, I will show that by measuring the entropy of each individuals trajectory, we find can explore the underlying predictability of human mobility, raising fundamental questions on how predictable we really are. I will also discuss the interplay between human mobilty, social links, and the predictive power of data mining.
\end{abstract}

